University of Wollongong

Research Online

Faculty of Engineering and Information

Faculty of Engineering and Information

Sciences - Papers: Part A

Sciences

2002

The dual reciprocity boundary element method for magnetohydrodynamic channel flows

Song-Ping Zhu

University of Wollongong, spz@uow.edu.au

Huan-Wen Liu

Guangxi University for Nationalities

Follow this and additional works at: https://ro.uow.edu.au/eispapers

Part of the Engineering Commons, and the Science and Technology Studies Commons

Research Online is the open access institutional repository for the University of Wollongong. For further information contact the UOW Library: research-pubs@uow.edu.au 


\title{
The dual reciprocity boundary element method for magnetohydrodynamic channel flows
}

\begin{abstract}
In this paper, we consider the problem of the steady-state fully developed magnetohydrodynamic (MHD) flow of a conducting fluid through a channel with arbitrary wall conductivity in the presence of a transverse external magnetic field with various inclined angles. The coupled governing equations for both axial velocity and induced magnetic field are firstly transformed into decoupled Poisson-type equations with coupled boundary conditions. Then the dual reciprocity boundary element method (DRBEM) [20] is used to solve the Poisson-type equations. As testing examples, flows in channels of three different crosssections, rectangular, circular and triangular, are calculated. It is shown that solutions obtained by the DRBEM with constant elements are accurate for Hartmann number up to 8 and for large conductivity parameters comparing to exact solutions and solutions by the finite element method (FEM).
\end{abstract}

\section{Keywords}

flows, method, dual, element, boundary, channel, magnetohydrodynamic, reciprocity

\section{Disciplines}

Engineering | Science and Technology Studies

\section{Publication Details}

Zhu, S. \& Liu, H. (2002). The dual reciprocity boundary element method for magnetohydrodynamic channel flows. The Anziam Journal, 44 305-322. 


\title{
THE DUAL RECIPROCITY BOUNDARY ELEMENT METHOD FOR MAGNETOHYDRODYNAMIC CHANNEL FLOWS
}

\author{
HUAN-WEN LIU ${ }^{1}$ and SONG-PING ZHU²
}

(Received 7 August, 1998; revised 15 July, 2001)

\begin{abstract}
In this paper, we consider the problem of the steady-state fully developed magnetohydrodynamic (MHD) flow of a conducting fluid through a channel with arbitrary wall conductivity in the presence of a transverse external magnetic field with various inclined angles. The coupled governing equations for both axial velocity and induced magnetic field are firstly transformed into decoupled Poisson-type equations with coupled boundary conditions. Then the dual reciprocity boundary element method (DRBEM) [20] is used to solve the Poisson-type equations. As testing examples, flows in channels of three different crosssections, rectangular, circular and triangular, are calculated. It is shown that solutions obtained by the DRBEM with constant elements are accurate for Hartmann number up to 8 and for large conductivity parameters comparing to exact solutions and solutions by the finite element method (FEM).
\end{abstract}

\section{Introduction}

The problem of magnetohydrodynamic (MHD) flow through channels has become important because of its practical applications in nuclear reactors, MHD flow meters, MHD generators, blood flow measurements, pumps, accelerators, and so on. Due to the coupling of equations of fluid mechanics and electrodynamics, exact solutions are out of the question for most practical cases. Therefore, it is always desirable to explore for more efficient numerical methods which render accurate numerical solutions.

In [25, 26], Singh and Lal have obtained numerical solutions of steady-state MHD flows through channels of triangular cross-sections by using a finite difference method (FDM) together with the Kantorovich technique [11]. Then, to overcome the drawback

\footnotetext{
${ }^{1}$ Department of Mathematics and Computer Science, Guangxi University for Nationalities, Nanning, Guangxi 530006, P.R. China; e-mail: huanwenliu@ hotmail.com.

${ }^{2}$ School of Mathematics and Applied Statistics, The University of Wollongong, Wollongong, NSW 2522, Australia; e-mail: spz@uow.edu.au.

(C) Australian Mathematical Society 2002, Serial-fee code 1446-8735/02
} 
of the finite difference mesh in fitting arbitrary cross-sections of the channel, they also presented a finite element method (FEM) to solve steady-state [27, 28] and unsteadystate [29] MHD channel flow problems with arbitrary wall conductivity. But with linear elements in the FEM they could only obtain results, at most, up to Hartmann number $M=5$. Later Tezer-Sezgin and Koksal [33] improved the FEM results for high Hartmann numbers by using quadratic elements for both velocity and magnetic fields. To further improve the accuracy of the FEM results, Gardner and Gardner [6] employed bi-cubic B-spline elements. However, their method is unavailable for arbitrary cross-sections of the channel.

To improve the computational efficiency, Tezer-Sezgin [30] and Tezer-Sezgin and Dost $[31,32]$ adopted a boundary element method (BEM). In their papers, both the coupled velocity and magnetic field equations are simplified into decoupled homogeneous or inhomogeneous modified Helmholtz equations with coupled boundary conditions and then solved by the conventional BEM [30,31] or by using radial basis functions [32].

However, one of the common problems appearing in all of the above mentioned works is the imposed magnetic field being generally assumed to be perpendicular to the direction of gravitational acceleration. Such an assumption simplified their models and numerical calculations. However, it also significantly reduced the versatility of their models.

In this paper, we present a more general case, in which the assumption that the imposed magnetic field is perpendicular to the direction of gravitational acceleration is no longer necessary. With an inclined imposed magnetic field, the coupled velocity and magnetic field equations are firstly transformed into two decoupled Poisson equations with coupled boundary conditions, and then solved by the dual reciprocity boundary element method (DRBEM) $[15,20]$. To show the accuracy of the DRBEM, three different geometries, that is, the rectangle, circle and triangle, are taken as the cross-section of the channel whose walls are arbitrarily conducting. Comparison is made between DRBEM solutions and other solutions, including exact solutions and numerical solutions obtained by using the FEM. Through the comparison, it is found that the agreement between the DRBEM solutions and other solutions is good for Hartmann numbers up to 8 although only the simplest constant elements in the DRBEM are used.

\section{Governing differential equations}

Let $\Omega$ represent the cross-section of a channel, through which a conducting fluid subject to a constant magnetic field flows, and $\Gamma$ be the boundary of $\Omega$. A Cartesian coordinate is chosen such that the $x y$-plane lies with a cross-section of the channel 
and the $z$ direction points to the direction along which the fluid flows. The magnetic field of strength $M$ is applied in the direction lying in the $x y$-plane but forming an angle $\phi$ with the $y$-axis.

It is well-known that Maxwell equations of electromagnetism and the basic equations of fluid mechanics lead to the coupled system of equations in the velocity and magnetic field. These equations for a viscous and incompressible fluid can be put in the following non-dimensional form [24]:

$$
\begin{aligned}
& \nabla^{2} V+M_{x} \frac{\partial B}{\partial x}+M_{y} \frac{\partial B}{\partial y}=-1, \\
& \nabla^{2} B+M_{x} \frac{\partial V}{\partial x}+M_{y} \frac{\partial V}{\partial y}=0,
\end{aligned}
$$

where

$$
\begin{gathered}
V=V_{z}\left(-\frac{b^{2}}{v \rho} \frac{d p}{d z}\right)^{-1}, \quad M_{x}=B_{0 x} b\left(-\frac{\sigma}{v \rho}\right)^{1 / 2}=M \sin \phi, \\
B=B_{z}\left(-\frac{b^{2}}{v \rho} \frac{d p}{d z} \mu_{0}(\nu \rho \sigma)^{1 / 2}\right)^{-1}, \quad M_{y}=B_{0 y} b\left(-\frac{\sigma}{v \rho}\right)^{1 / 2}=M \cos \phi, \\
M=\left(M_{x}^{2}+M_{y}^{2}\right)^{1 / 2}=B_{0} b(-\sigma / v \rho)^{1 / 2},
\end{gathered}
$$

and $\nu, \rho$ and $\sigma$ are, respectively, the kinematic viscosity, density and electric conductivity of the fluid, $\mu_{0}$ is the magnetic permeability in vacuum, $d p / d z$ is the constant axial pressure gradient, $B_{0}$ is the strength of the applied magnetic field, $B_{0 x}$ and $B_{0 y}$ are respectively the $x$ and $y$ components of the applied magnetic field, $V_{z}$ and $B_{z}$ are respectively the $z$ components of velocity and induced magnetic field. Here $V, B$ and $M$ are called axial velocity, induced magnetic field and the Hartmann number, respectively.

A general form of the boundary conditions can be written as

$$
\begin{array}{ll}
V=0 & \text { on } \Gamma, \\
\frac{\partial B}{\partial \mathbf{n}}+\lambda B=0 & \text { on } \Gamma,
\end{array}
$$

where $\mathbf{n}$ is the outward normal unit vector of the boundary and $\lambda$ is the conductivity parameter given by $\lambda=\sigma a /\left(\sigma^{\prime} h\right)$, where $a$ is a characteristic length, $\sigma$ and $\sigma^{\prime}$ are the electrical conductivity of the fluid and of the walls, respectively, and $h$ is the thickness of the walls.

There are two special cases as far as the wall conductivity is concerned. For a non-conducting wall, $\sigma^{\prime}=0$ or $\lambda=\infty$, condition (3) reduces to $B=0$ on $\partial \Omega$. On the other hand, for a perfectly conducting wall, $\sigma^{\prime}=\infty$ or $\lambda=0$, condition (3) becomes $\partial B / \partial \mathbf{n}=0$ on $\partial \Omega$. 
Equations (1) may be decoupled by the introduction of two new variables $W_{1}$ and $W_{2}$ defined as $W_{1}=V+B$ and $W_{2}=V-B$. Under these new variables, the decoupled differential system now has the form

$$
\begin{aligned}
& \nabla^{2} W_{1}+M_{x} \frac{\partial W_{1}}{\partial x}+M_{y} \frac{\partial W_{1}}{\partial y}=-1, \\
& \nabla^{2} W_{2}-M_{x} \frac{\partial W_{2}}{\partial x}-M_{y} \frac{\partial W_{2}}{\partial y}=-1,
\end{aligned}
$$

subject to the coupled boundary conditions

$$
W_{1}=-W_{2}, \quad \frac{\partial W_{1}}{\partial \mathbf{n}}+\lambda W_{1}=\frac{\partial W_{2}}{\partial \mathbf{n}}+\lambda W_{2} .
$$

Furthermore, if we set $u_{1}=W_{1} e^{\left(x M_{x}+y M_{y}\right) / 2}$ and $u_{2}=W_{2} e^{-\left(x M_{x}+y M_{y}\right) / 2}$, (4) can then be rewritten as

$$
\nabla^{2} u_{1}=\frac{M^{2}}{4} u_{1}-e^{\left(x M_{x}+y M_{y}\right) / 2}, \quad \nabla^{2} u_{2}=\frac{M^{2}}{4} u_{2}-e^{-\left(x M_{x}+y M_{y}\right) / 2},
$$

subject to the boundary conditions

$$
\begin{aligned}
u_{1} & =-u_{2} e^{x M_{x}+y M_{y}}, \\
\frac{\partial u_{1}}{\partial \mathbf{n}}+\lambda u_{1}-\frac{u_{1}}{2}\left(M_{x}, M_{y}\right) \cdot \mathbf{n} & =\left(\frac{\partial u_{2}}{\partial \mathbf{n}}+\lambda u_{2}+\frac{u_{2}}{2}\left(M_{x}, M_{y}\right) \cdot \mathbf{n}\right) e^{x M_{x}+y M_{y}},
\end{aligned}
$$

or

$$
u_{1}=-u_{2} e^{x M_{x}+y M_{y}}, \quad \frac{\partial u_{1}}{\partial \mathbf{n}}=\left(\frac{\partial u_{2}}{\partial \mathbf{n}}+2 \lambda u_{2}\right) e^{x M_{x}+y M_{y}} .
$$

The boundary conditions (6) can be decoupled only for a special case when the boundary wall is non-conducting. Because of this, analytical solution is generally unavailable and thus numerical solutions must be resorted to. Therefore, it is always desirable to search for more efficient as well as accurate numerical methods. It is worth indicating that (5) are in fact the modified Helmholtz equations with an inhomogeneous term. Tezer-Sezgin and Dost [32] solved them by using radial basis functions. In this paper, we shall treat (5) as Poisson equations and solve them by using the DRBEM $[4,20,36]$.

\section{The dual reciprocity boundary element method}

The BEM is now a well-established numerical technique for solving boundary-value problems that involve linear as well as certain types of nonlinear partial differential 
equations (PDEs) [1]. The basic idea of the technique is to find an integral equation equivalent to the original PDE, and to solve this integral equation using a discretisation procedure as with any other numerical approach. For certain types of linear and homogeneous PDE, only a boundary discretisation is necessary; this reduction in the dimensionality of the problem permits accurate solutions to be obtained very efficiently, and is the main attraction of the BEM approach. However, for an inhomogeneous PDE, the integral equation involves a domain integral, and the reduction in dimensionality is apparently lost. Unfortunately, the Poisson-type equations (5) to be dealt with in this paper fall into this category.

Among various methods for converting domain integrals into boundary integrals in the BEM for inhomogeneous PDEs, the most successful one is the so-called DRBEM, which was first proposed by Nardini and Brebbia [15] in 1982 and later improved by many others [19, 21, 40, 35]. While many variations, such as the Laplace transform dual reciprocity method (LTDRM) [38, 37, 39], the separation of variables-dual reciprocity method (SOVDRM) [3] and the perturbation DRBEM [12, 13], have been proposed since its birth, the DRBEM is still evolving and many researchers are currently actively involved in this area of research. The main idea of the DRBEM is to divide the solution into two parts: a known particular solution of the inhomogeneous PDE plus a complementary solution of its homogeneous counterpart. Since particular solutions to complex problems are very difficult or sometimes even impossible to obtain, the inhomogeneity is approximated by a series of simpler radial basis functions (RBFs) for which particular solutions can be easily determined.

For the completeness of the current paper, we shall briefly describe the DRBEM here. First of all, both terms on the right-hand side of (5) are expanded as a series of interpolation functions $f_{j}(\mathbf{x})=f_{j}(x, y)$, that is,

$$
\frac{M^{2}}{4} u_{1}-e^{\left(x M_{x}+y M_{y}\right) / 2} \approx \sum_{j=1}^{n} \alpha_{j}^{(1)} f_{j}(\mathbf{x}), \quad \frac{M^{2}}{4} u_{2}-e^{-\left(x M_{x}+y M_{y}\right) / 2} \approx \sum_{j=1}^{n} \alpha_{j}^{(2)} f_{j}(\mathbf{x}),
$$

where $\alpha_{j}^{(1)}$ and $\alpha_{j}^{(2)}$ are the coefficients to be determined by the following interpolation conditions at $n=N+L$ collocation points $\mathbf{x}_{i}=\left(x_{i}, y_{i}\right)$ :

$$
b_{i}^{(k)}=\sum_{j=1}^{n} \alpha_{j}^{(k)} f_{j}\left(\mathbf{x}_{i}\right), \quad i=1, \ldots, n, \quad k=1,2,
$$

where

$$
b_{i}^{(1)}=\frac{M^{2}}{4} u_{1}\left(x_{i}, y_{i}\right)-e^{\left(x_{i} M_{x}+y_{i} M_{y}\right) / 2}, \quad b_{i}^{(2)}=\frac{M^{2}}{4} u_{2}\left(x_{i}, y_{i}\right)-e^{-\left(x_{i} M_{x}+y_{i} M_{y}\right) / 2} .
$$

There are many ways of choosing the interpolation functions $f_{j}$. Partridge and Brebbia [19] showed that satisfactory results can usually be obtained if $f_{j}$ takes the form $\sum_{m=0}^{s}\left\|\mathbf{x}-\mathbf{x}_{j}\right\|^{m}$. Moreover, they also pointed out that the use of $s=1$ 
giving $f_{j}=1+\left\|\mathbf{x}-\mathbf{x}_{j}\right\|$ is generally sufficient. Then Duchon [5] demonstrated that the so-called augmented thin plate splines (ATPS), which are a combination of the thin plate splines (TPS), $\left\|\mathbf{x}-\mathbf{x}_{j}\right\|^{2} \log \left\|\mathbf{x}-\mathbf{x}_{j}\right\|$, and some augmented linear terms $1, x, y$ in $\mathbf{R}^{2}$ (1, $x, y, z$ in $\mathbf{R}^{3}$ ), are the optimal interpolation functions in two and three dimensional spaces respectively in the sense that they interpolate $f$ in $\mathbf{R}^{d}$ $(d=2,3)$ with a minimised rotation-invariant seminorm. This theoretical work has been strongly supported by some numerical results [2, 7, 38]. However, despite their optimal properties, ATPS have some drawbacks. For example, they are only $C^{1}$ in $\mathbf{R}^{2}$ and non-differentiable in $\mathbf{R}^{3}$ and their convergence is slow (see [14, 22, 34]). Recently, some researchers $[8,37]$ suggested that the family of multiquadrics $\varphi_{\beta}\left(\left\|\mathbf{x}-\mathbf{x}_{j}\right\|\right)=$ $\left(c^{2}+\left\|\mathbf{x}-\mathbf{x}_{j}\right\|^{2}\right)^{\beta / 2}$ can overcome the above drawbacks of ATPS. Here $\beta$ is an odd integer and $c$ is a parameter. However, others argued that the choice of the parameter $c$ still could affect the accuracy of the solution by several orders of magnitude [8]. For the sake of simplicity, we shall still adopt the simplest RBF $1+\left\|\mathbf{x}-\mathbf{x}_{j}\right\|$ in this paper.

The $n$ collocation points $\mathbf{x}_{i}, i=1, \ldots, n$, consist of $N$ boundary collocation points of the BEM and $L$ internal nodes. To ensure the accuracy of the DRBEM solution, some internal nodes normally have to be included. However, a new technique without any internal collocation point, called the Multiple Reciprocity Boundary Element Method (MRBEM), has been recently developed by Nowak and Brebbia and applied to solve the Poisson equation [17] and the Helmholtz equation [18]. Then Neves and Brebbia [16] and Itagaki and Brebbia [10] separately extended it to solve the Navier equations of elasticity and the modified Helmholtz equations. The MRBEM can be thought of as an extension of the idea of the DRBEM. However, instead of approximating the source term by the set of RBFs, a sequence of functions related to the fundamental solution is introduced. These functions constitute a set of higher order fundamental solutions which permit the second Green's identity to be applied to each term of the sequence. As a result, the MRBEM leads, in the limit, to the exact boundary only formulation of the domain integrals and therefore no internal collocation points are needed. Indeed, the current problem may be solved by adopting the MRBEM too if we treat (5) as inhomogeneous modified Helmholtz equations. But this is beyond the scope of our current research and thus should not be discussed here.

System (7) can be written in matrix form as

$$
\left[\begin{array}{c}
b_{1}^{(i)} \\
b_{2}^{(i)} \\
\vdots \\
b_{n}^{(i)}
\end{array}\right]=\left[\begin{array}{cccc}
f_{11} & f_{12} & \cdots & f_{1 n} \\
f_{21} & f_{22} & \cdots & f_{2 n} \\
\vdots & \vdots & \ddots & \vdots \\
f_{n 1} & f_{n 2} & \cdots & f_{n n}
\end{array}\right]\left[\begin{array}{c}
\alpha_{1}^{(i)} \\
\alpha_{2}^{(i)} \\
\vdots \\
\alpha_{n}^{(i)}
\end{array}\right] \quad \text { or } \quad \mathbf{b}^{(i)}=\mathbf{F} \boldsymbol{\alpha}^{(i)}, \quad i=1,2 .
$$

The coefficients $\boldsymbol{\alpha}^{(i)}$ can then be found simply by inverting the matrix in (8) to produce

$$
\boldsymbol{\alpha}^{(i)}=\mathbf{F}^{-1} \mathbf{b}^{(i)}, \quad i=1,2 .
$$


Let $u^{*}$ denote the fundamental solution of the Laplace equation:

$$
u^{*}(\mathbf{x})=\frac{1}{2 \pi} \ln \frac{1}{\|\mathbf{x}-\xi\|},
$$

which satisfies $\nabla^{2} u=-\delta(\mathbf{x}-\boldsymbol{\xi})$. Applying the usual boundary element technique, (5) are now multiplied by the fundamental solution $u^{*}$ and integrated over the domain $\Omega$ to yield

$$
\int_{\Omega}\left(\nabla^{2} u_{i}\right) u^{*} d \Omega=\sum_{j=1}^{n} \alpha_{j}^{(i)} \int_{\Omega} f_{j} u^{*} d \Omega, \quad i=1,2 .
$$

Now, integrating by parts in (10) produces, with (8) being utilised,

$$
c_{\xi} u_{i \xi}-\int_{\Gamma}\left(u^{*} q_{i}-q^{*} u_{i}\right) d \Gamma=\sum_{j=1}^{n} \alpha_{j}^{(i)}\left(c_{\xi} \hat{u}_{\xi j}-\int_{\Gamma}\left(u^{*} \hat{q}_{j}-q^{*} \hat{u}_{j}\right)\right) d \Gamma, \quad i=1,2,
$$

where $\hat{u}_{j}=\left\|\mathbf{x}-\mathbf{x}_{j}\right\|^{2} / 4+\left\|\mathbf{x}-\mathbf{x}_{j}\right\|^{3} / 9, j=1, \ldots, n$, is a particular solution of the equation $\nabla^{2} u=f_{j}$ and $\xi$ is a source point of $u^{*}$ which can be any point within the domain or on the boundary; $u_{\xi}$ and $\hat{u}_{\xi j}$ are the values of $u$ and $\hat{u}_{j}$ at point $\xi$, respectively; and $q_{1}, q_{2}, q^{*}$ and $\hat{q}_{j}$ are the normal derivatives of $u_{1}, u_{2}, u^{*}$ and $\hat{u}_{j}$, respectively. The term $c_{\xi}$ in (11) depends upon the location of the source point $\xi$ :

$$
c_{\xi}= \begin{cases}\alpha(\xi) / 2 \pi, & \text { if } \xi \text { is a boundary point of } \Omega ; \\ 1, & \text { if } \xi \text { is an interior point of } \Omega,\end{cases}
$$

where $\alpha(\xi)$ denotes the internal angle of the boundary at source point $\xi$. If we adopt constant boundary elements, the discretised form of (11) can be written as

$$
c_{\xi} u_{i \xi}-\sum_{k=1}^{N}\left(G_{\xi k} q_{i k}-\hat{H}_{\xi k} u_{i k}\right)=\sum_{j=1}^{n} \alpha_{j}^{(i)}\left(c_{\xi} \hat{u}_{\xi j}-\sum_{k=1}^{N}\left(G_{\xi k} \hat{q}_{k j}-\hat{H}_{\xi k} \hat{u}_{k j}\right)\right), i=1,2 .
$$

Applying (12) to all collocation points, one obtains a linear system of equations

$$
\begin{aligned}
c_{i} u_{m i} & -\sum_{k=1}^{N}\left(G_{i k} q_{m k}-\hat{H}_{i k} u_{m k}\right) \\
& =\sum_{j=1}^{n} \alpha_{j}^{(m)}\left(c_{i} \hat{u}_{i j}-\sum_{k=1}^{N}\left(G_{i k} \hat{q}_{k j}-\hat{H}_{i k} \hat{u}_{k j}\right)\right), \quad i=1, \ldots, n, m=1,2,
\end{aligned}
$$

which can be written in matrix form as:

$$
\mathbf{H U}_{m}-\mathbf{G Q}_{m}=(\mathbf{H} \hat{\mathbf{U}}-\mathbf{G} \hat{\mathbf{Q}}) \boldsymbol{\alpha}^{(m)}, \quad m=1,2,
$$


with

$$
\begin{aligned}
& \mathbf{H}=\left(H_{i j}\right)_{n \times n}, \quad \mathbf{G}=\left(G_{i j}\right)_{n \times N}, \quad \mathbf{U}_{1}=\left(u_{11}, \ldots, u_{1 n}\right)^{T}, \quad \mathbf{Q}_{1}=\left(q_{11}, \ldots, q_{1 n}\right)^{T}, \\
& \hat{\mathbf{U}}=\left(\hat{u}_{i j}\right)_{n \times n}, \quad \hat{\mathbf{Q}}=\left(\hat{q}_{i j}\right)_{N \times n}, \quad \mathbf{U}_{2}=\left(u_{21}, \ldots, u_{2 n}\right)^{T}, \quad \mathbf{Q}_{2}=\left(q_{21}, \ldots, q_{2 n}\right)^{T},
\end{aligned}
$$

where $u_{i}, q_{i}, \hat{u}_{i j}$ and $\hat{q}_{i j}$ are the values of $u, q, \hat{u}_{j}$ and $\hat{q}_{j}$ at points $\mathbf{x}_{i}$, respectively, and

$$
H_{i j}= \begin{cases}\alpha\left(\mathbf{X}_{i}\right) /(2 \pi), & i=j=1, \ldots, N \\ 1, & i=j=N+1, \ldots, n \\ \hat{H}_{i j}, & i=1, \ldots, n, j=1, \ldots, N, i \neq j \\ 0, & i=1, \ldots, n, j=N+1, \ldots, n, i \neq j\end{cases}
$$

where the definitions of $\hat{H}_{i j}$ and $G_{i j}$ are as in [1].

Substituting (9) into (13), we obtain

or

$$
\mathbf{H} \mathbf{U}_{m}-\mathbf{G} \mathbf{Q}_{m}=(\mathbf{H} \hat{\mathbf{U}}-\mathbf{G} \hat{\mathbf{Q}}) \mathbf{F}^{-1} \mathbf{b}^{(m)}, \quad m=1,2,
$$

$$
\mathbf{H} \mathbf{U}_{m}-\mathbf{G Q}_{m}=\mathbf{S}\left(\frac{M^{2}}{4} \mathbf{U}_{m}-\mathbf{E}^{(m)}\right), \quad m=1,2,
$$

with $\mathbf{S}=(\mathbf{H} \hat{\mathbf{U}}-\mathbf{G} \hat{\mathbf{Q}}) \mathbf{F}^{-1}$ and

$$
\begin{aligned}
& \mathbf{E}^{(1)}=\left(e^{\left(x_{1} M_{x}+y_{1} M_{y}\right) / 2}, \ldots, e^{\left(x_{n} M_{x}+y_{n} M_{y}\right) / 2}\right)^{T}, \\
& \mathbf{E}^{(2)}=\left(e^{-\left(x_{1} M_{x}+y_{1} M_{y}\right) / 2}, \ldots, e^{-\left(x_{n} M_{x}+y_{n} M_{y}\right) / 2}\right)^{T} .
\end{aligned}
$$

So a final $2 n \times 2 n$ linear system of equations

$$
\left(\mathbf{H}-\frac{M^{2}}{4} \mathbf{S}\right) \mathbf{U}_{m}-\mathbf{G} \mathbf{Q}_{m}=-\mathbf{S E} \mathbf{E}^{(m)}, \quad m=1,2,
$$

is obtained. Upon imposing the boundary conditions (6), such a linear system can be readily solved. After all the unknown values $u_{i}$ or $q_{i}$ are found by solving the linear system (14), a desired solution for any interior point $\boldsymbol{\xi}$ can be evaluated from (12).

\section{Numerical examples}

In this section, as numerical examples for the DRBEM applied to solve MHD flow problems, three different cross-sections of the channel, that is, rectangular, circular and triangular, are considered. Though these three cross-sections of the channel are also studied by Singh and Lal using the FDM [25] (only the triangular case) and the 

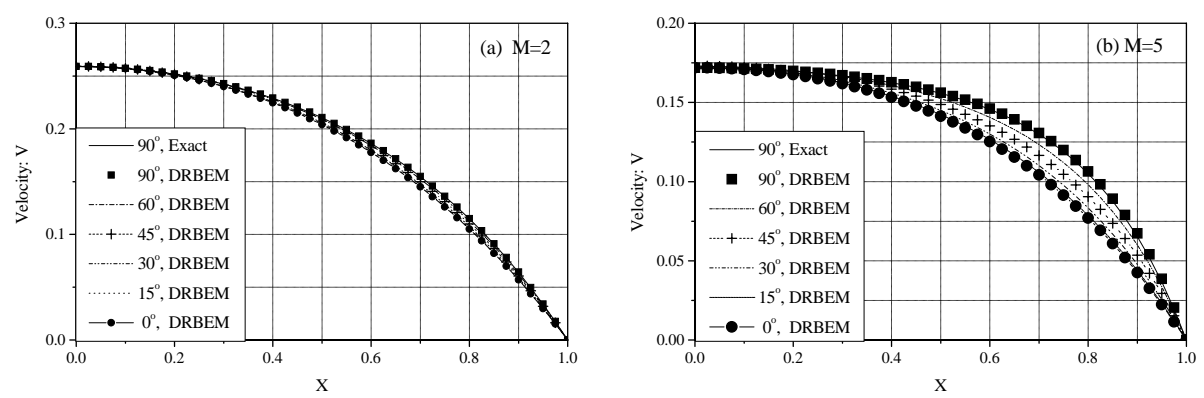

FIGURE 1. Profiles of the axial velocity along the $x$-axis of a rectangular channel in the presence of the magnetic field applied to the channel with various angles $\phi$ for a non-conducting wall; (a) $M=2$,

(b) $M=5$.
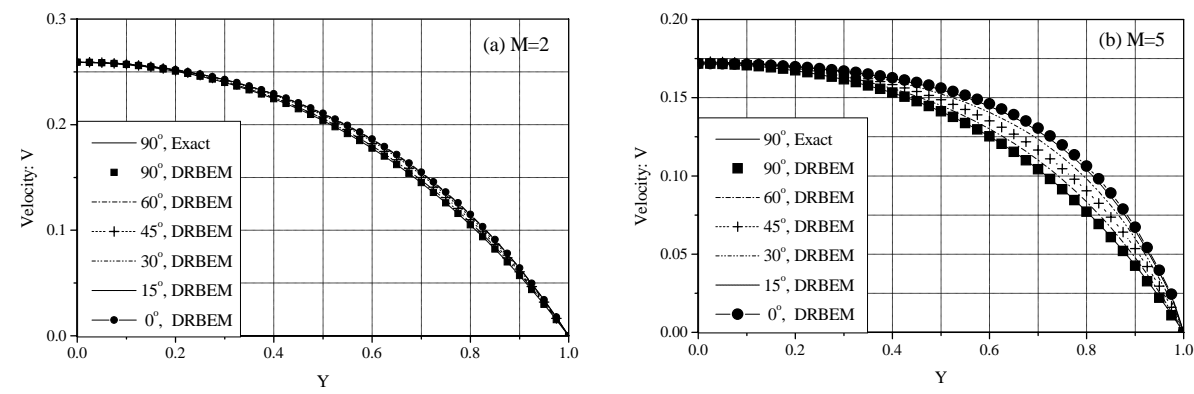

FIGURE 2. Profiles of the axial velocity along the $y$-axis of a rectangular channel in the presence of the magnetic field applied to the channel with various angles $\phi$ for a non-conducting wall; (a) $M=2$, (b) $M=5$.

FEM [27] (all three cases), the applied magnetic field is generally assumed to act in the direction being perpendicular to the $y$-axis only, that is, $\phi=\pi / 2$. In this paper, we are able to present more general cases; those problems subject to an inclined applied magnetic field with an arbitrary angle $\phi$ can now be calculated with the presence of both conducting and non-conducting walls.

4.1. Rectangular channel The cross-section of an infinitely long rectangular channel is oriented with its two perpendicular sides parallel to the $x$ - and $y$-axes, respectively and the origin of the coordinates at the geometric centre of the cross-section. Both the channel width and height are taken to be unity. For all the results presented in this section, 160 constant elements are used and 169 internal collocation points are evenly distributed in the interior of the rectangular domain. Here, we shall show the results of various cases.

Case (i): $\lambda=\infty$ and $\phi$ can be arbitrary. But we shall only present the results of six different $\phi$ values, $\phi=\pi / 2, \pi / 3, \pi / 4, \pi / 6, \pi / 12,0$. For $M=2,5$, DRBEM 

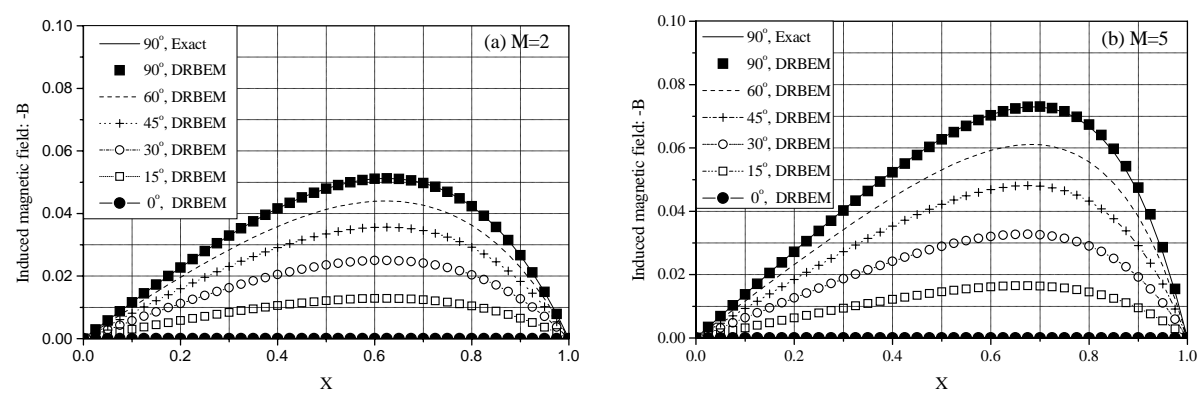

FIGURE 3. Profiles of the induced magnetic field $-B$ along the $x$-axis of a rectangular channel in the presence of the magnetic field applied to the channel with various angles $\phi$ for a non-conducting wall; (a) $M=2$, (b) $M=5$.
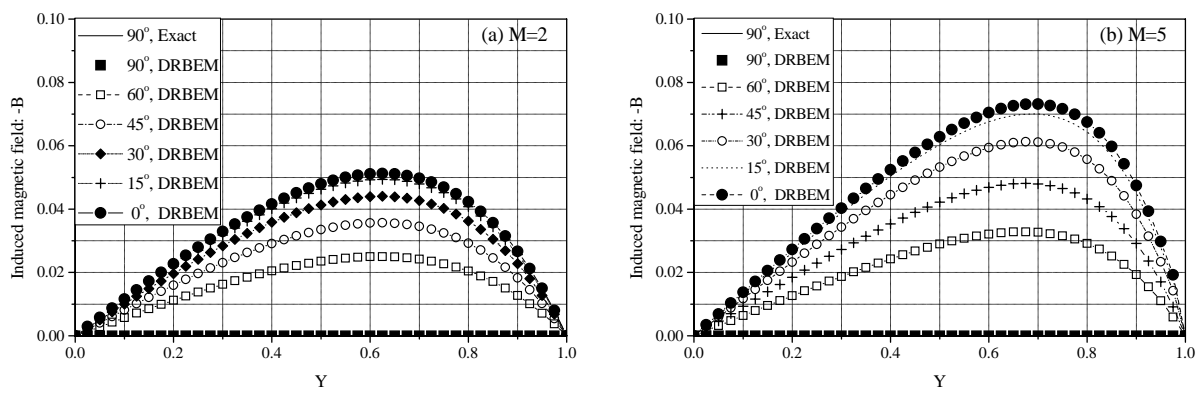

FIGURE 4. Profiles of the induced magnetic field $-B$ along the $y$-axis of a rectangular channel in the presence of the magnetic field applied to the channel with various angles $\phi$ for a non-conducting wall; (a) $M=2$, (b) $M=5$.

solutions of the axial velocity along the $x$ - and $y$-axes are plotted in Figures 12 respectively and DRBEM solutions of the corresponding induced magnetic field along the $x$ - and $y$-axes are plotted in Figures 3-4. In the case of $\phi=\pi / 2$, the exact solutions from Shercliff [24] are also plotted. As we can see in Figures 1-2, when the applied magnetic field inclines more towards the $y$-axis, that is, $\phi$ decreases, the axial velocity decreases slightly along the $x$-axis but increases slightly along the $y$-axis. A similar behaviour can be observed in Figures 3-4 for the induced magnetic field $-B$. However, the changes in axial velocity are not significant in comparison with those changes in the induced magnetic field although the changes in axial velocity do become more noticeable when $M$ is increased from 2 to 5 .

Case (ii): $\lambda$ is arbitrary but $\phi$ is fixed to $\pi / 2$. The axial velocity and the induced magnetic field along the $x$-axis are calculated for conducting walls with $\lambda=1,10$ and $\infty$ when $M=2,5$, respectively. A comparison between the present results and FEM results [28] is made and plotted in Figures 5-6. Exact solutions for non-conducting 

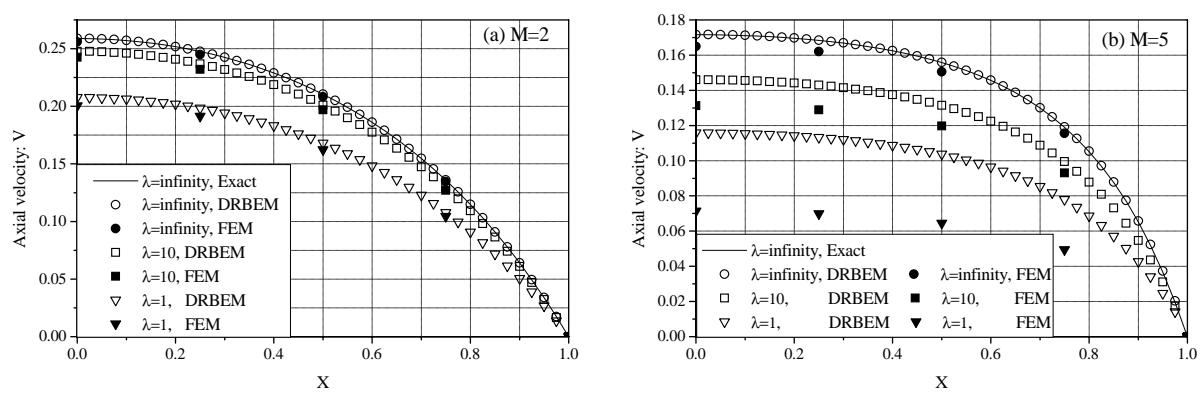

FIGURE 5. The present DRBEM solutions and FEM solutions [28] of the axial velocity $V$ along the $x$-axis for a conducting wall with $\phi=\pi / 2$; (a) $M=2$, (b) $M=5$.
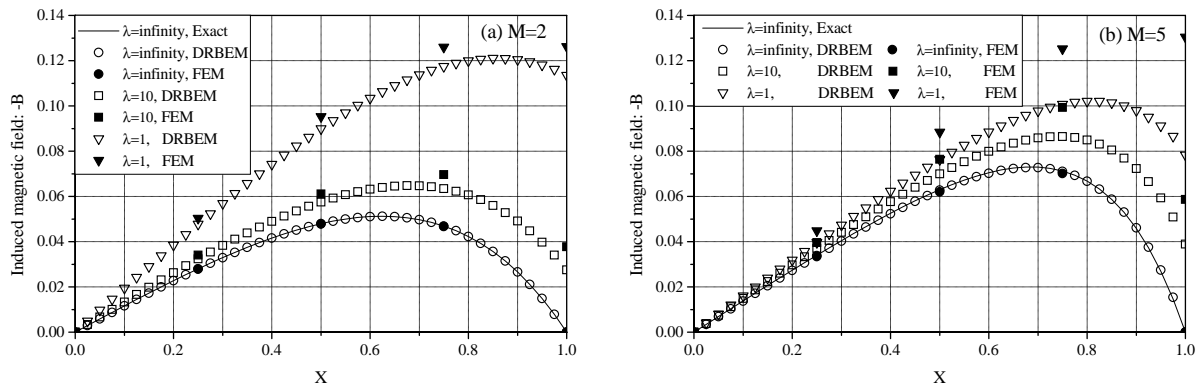

FIGURE 6. The present DRBEM solutions and FEM solutions [28] of the induced magnetic field $-B$ along the $x$-axis for a conducting wall with $\phi=\pi / 2$; (a) $M=2$, (b) $M=5$.
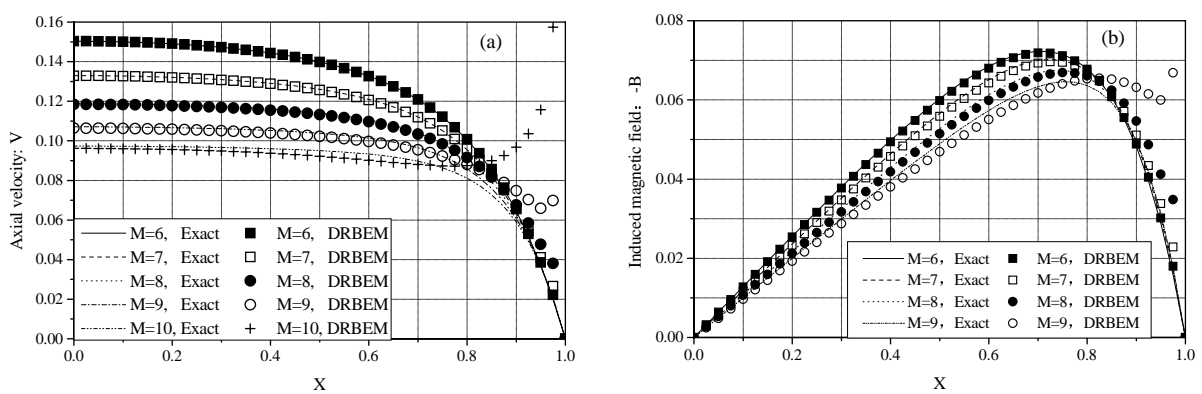

FIgURE 7. (a) Profiles of $V$ along the $x$-axis of the rectangular channel with $\phi=\pi / 2$ and $\lambda=\infty$ for $M=6,7,8,9,10$; (b) profiles of $-B$ along the $x$-axis of the rectangular channel with $\phi=\pi / 2$ and $\lambda=\infty$ for $M=6,7,8,9$. 

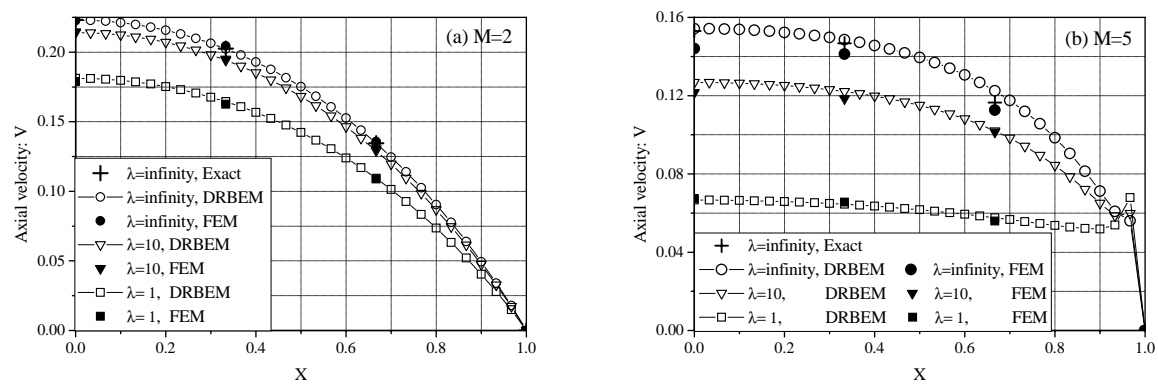

FIGURE 8 . Profiles of the axial velocity $V$ along the $x$-axis of a circular channel for $\lambda=1,10$ and $\infty$ and for $\phi=\pi / 2$; (a) $M=2$, (b) $M=5$.
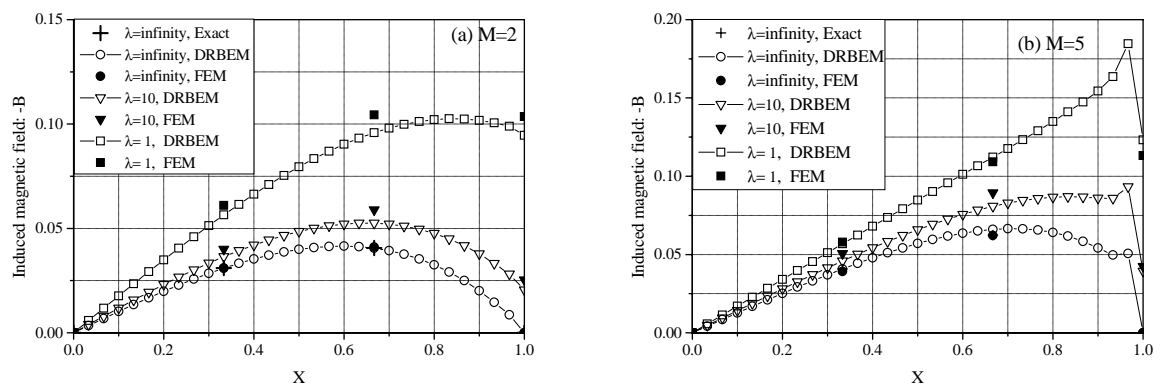

FIGURE 9. Profiles of the induced magnetic field $-B$ along the $x$-axis of a circular channel for $\lambda=1,10$ and $\infty$ and for $\phi=\pi / 2$; (a) $M=2$, (b) $M=5$.

walls from Shercliff [24] are also plotted in these figures to provide a comparison for the case when $\lambda=\infty$. Clearly our DRBEM solution offers excellent agreement with the exact solution when $\lambda=\infty$. Such an excellent agreement for the case when $\lambda=\infty$ also gives us confidence in the accuracy level of our results for other cases where there are no analytical solutions to compare with. The FEM results obtained by Singh and Lal [28] seem to have underestimated the axial velocity while overestimating the induced magnetic field along the $x$-axis as shown in Figures 5-6. In fact, such a disagreement between our current solutions and the FEM solutions worsens when $M$ is larger and $\lambda$ is smaller.

It is also interesting to observe that the axial velocity increases with the increased conductivity parameter $\lambda$, whereas the induced magnetic field $-B$ behaves in a quite opposite way; at every $x$ point, the strength of the induced magnetic field $-B$ decreases as $\lambda$ increases.

Finally, the DRBEM is tested for relatively high Hartmann numbers $M=6,7$, 8, 9 and 10. Profiles of both the axial velocity and the induced magnetic field along the $x$-axis for the non-conducting wall from the DRBEM and from Shercliff [24] are 

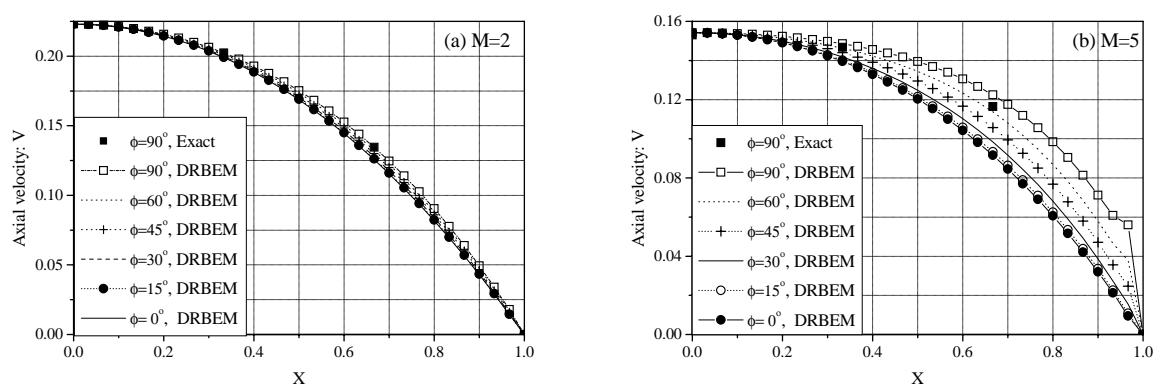

FIGURE 10. Profiles of the axial velocity $V$ along the $x$-axis of a circular channel in the presence of the magnetic field applied to the channel with various angles $\phi$; (a) $M=2$, (b) $M=5$.
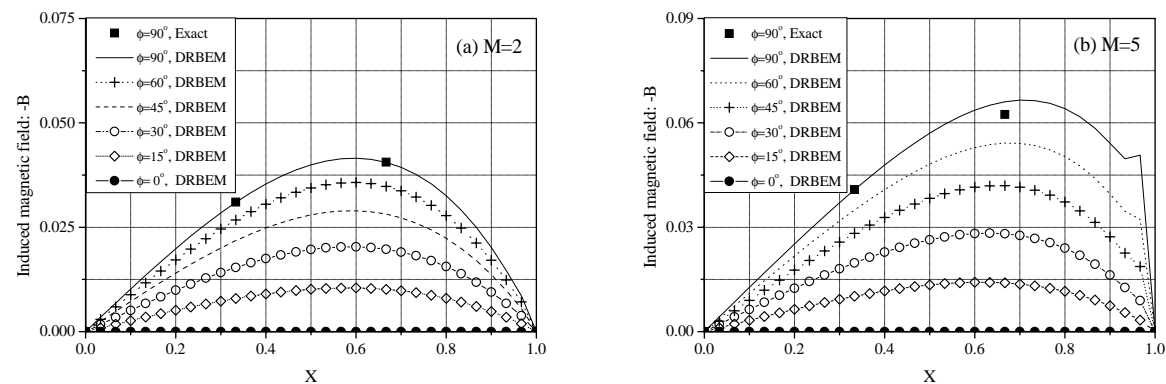

FIGURE 11. Profiles of the induced magnetic field $-B$ along the $x$-axis of a circular channel in the presence of the magnetic field applied to the channel with various angles $\phi$; (a) $M=2$, (b) $M=5$.

plotted in Figure 7. As we can see, the DRBEM is accurate for Hartmann number up to 8. If $M$ is increased beyond 8 , the error near the boundary reaches an intolerable level. This suggests that the adoption of high-order elements such as linear and quadratic elements may become necessary for high Hartmann numbers.

4.2. Circular channel When the cross-section of a channel is circular, we place the origin of the coordinate system at the geometric centre of the cross-section and take the radius as the characteristic length. In our calculation, 60 boundary elements were used and 64 internal collocation points were evenly distributed on four inner circles with the radii being $1 / 3,1 / 2,2 / 3$ and $14 / 15$, respectively.

In order to compare with Singh and Lal's FEM solution [28], we calculated the axial velocity and the induced magnetic field along the positive $x$-axis using the present DRBEM for $\lambda=1,10$ and $\infty$ when $M=2,5$, respectively, for a fixed $\phi=\pi / 2$. The results are plotted in Figures 8-9. As can be seen from these figures, the overall agreement between the present DRBEM results and the FEM results is very good. However, as far as the velocity field is concerned, the difference between these two sets of results becomes slightly larger when $\lambda=\infty$ for the case of $M=5$. On the 

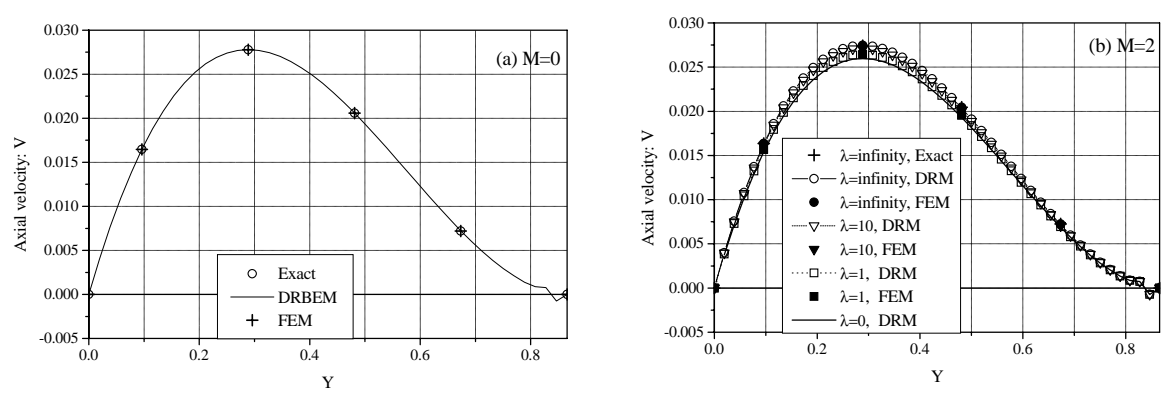

FIGURE 12. Profiles of the axial velocity $V$ along the $y$-axis of a triangular channel for arbitrary wall conductivity and $\phi=\pi / 2$; (a) $M=0$, (b) $M=2$.
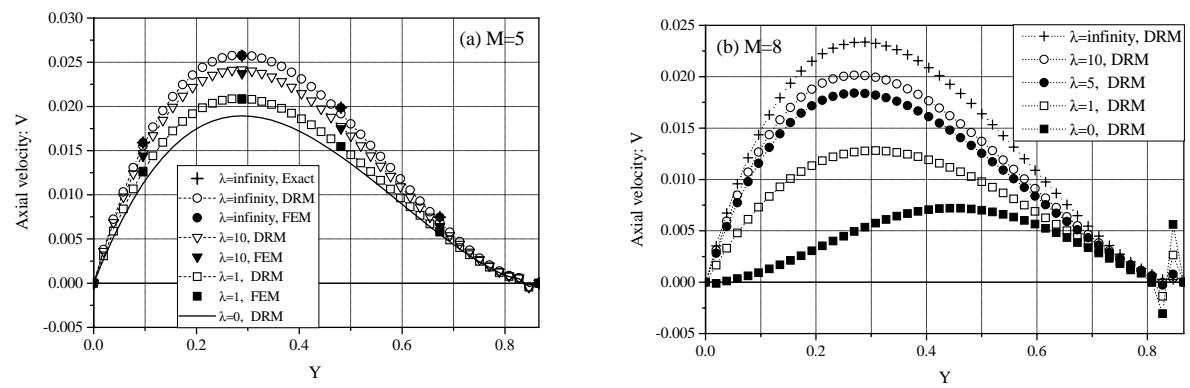

FIGURE 13. Profiles of the axial velocity $V$ along the $y$-axis of a triangular channel for arbitrary wall conductivity and $\phi=\pi / 2$; (a) $M=5$, (b) $M=8$.

other hand, the major difference, although not very large, between the two sets of results for the induced magnetic field along the positive $x$-axis is mainly observed for the case of a smaller $M$ value and small $\lambda$ as shown in Figure 9.

In the case of a non-conducting wall, the exact solution is available in [9] and is also plotted. It is shown that the agreement between the present DRBEM, the FEM and the exact solution is good. In addition, for a non-conducting wall, different angles of inclination of the applied magnetic field with $\phi$ being $\pi / 2, \pi / 3, \pi / 4, \pi / 6, \pi / 12$ and 0 are also considered. We see from Figures $10-11$ that both the axial velocity $V$ and the induced magnetic field $-B$ along the $x$-axis decrease with the applied magnetic field inclining towards the $y$-axis (that is, $\phi$ decreasing), respectively. For $M=2$, the velocity distribution is not sensible to the change of the inclination angle of the applied magnetic field at all, whereas when $M$ is increased to a larger value $(M=5)$, a larger inclination angle makes a significantly larger downstream velocity.

4.3. Equilateral triangular channel Now, with a channel of triangular crosssection, we place the origin of the coordinate system at the mid-point of the base and the $x$-axis along the base with the length of a side as the characteristic length. 

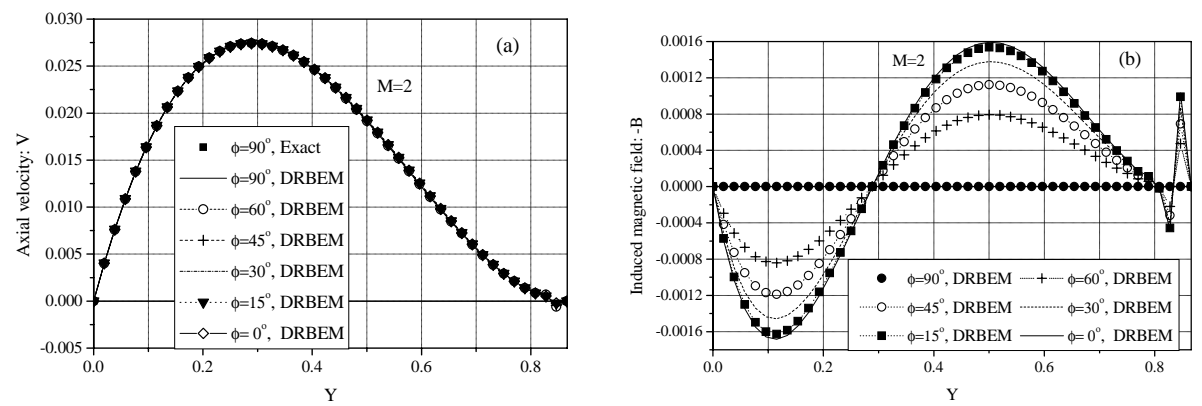

FIGURE 14. Profiles of (a) the axial velocity $V$ and (b) the induced magnetic field $-B$ along the $y$-axis of a triangular channel in the presence of the magnetic field applied to the channel with various angles $\phi$ for $\lambda=\infty$ and $M=2$.
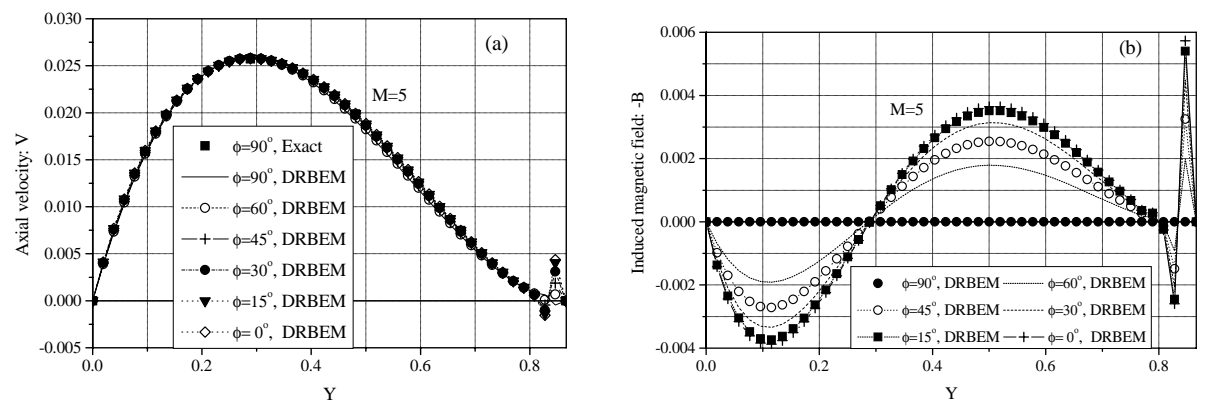

FIGURE 15. Profiles of (a) the axial velocity $V$ and (b) the induced magnetic field $-B$ along the $y$-axis of a triangular channel in the presence of the magnetic field applied to the channel with various angles $\phi$ for $\lambda=\infty$ and $M=5$.
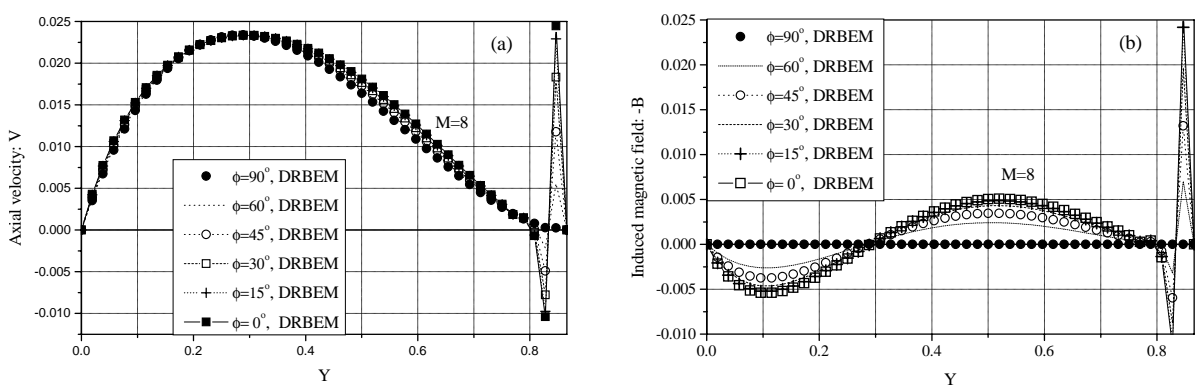

FIGURE 16. Profiles of (a) the axial velocity $V$ and (b) the induced magnetic field $-B$ along the $y$-axis of a triangular channel in the presence of the magnetic field applied to the channel with various angles $\phi$ for $\lambda=\infty$ and $M=8$. 
The boundary of $\Omega$ is then defined by the lines $y=0, y= \pm \sqrt{3}(x \pm 0.5)$. In the present method, we used 60 boundary elements and 28 internal collocation points. The distribution of the 28 internal collocation points is the same as that in [27]. For a fixed $\phi(\phi=\pi / 2)$ and four different $M$ values ( $M=0,2,5$ and 8$)$, the results of the axial velocity along the $y$-axis for $\lambda=0,1,10, \infty$ obtained from the present DRBEM model are compared to FEM solutions [28] and exact solutions [28] (only for $\lambda=\infty$ ) in Figures 12-13. It is interesting to have observed from these figures that with small Hartmann numbers (for example $M=2$ ), the axial velocity does not seem to change very much when the wall conductivity parameter $\lambda$ varies from one extreme $(\lambda=0)$ to another $(\lambda=\infty)$.

In the case of a non-conducting wall, the axial velocity and the induced magnetic field along the $y$-axis for the inclined applied magnetic field with $\phi$ being $\pi / 2, \pi / 3$, $\pi / 4, \pi / 6, \pi / 12$ and 0 are also calculated for $M=2,5$ and 8 . It is found from Figures 14-16 (a) that the effect of the inclination angle $\phi$ on the axial velocity is not significant. However, the induced magnetic field $-B$ along the $y$-axis increases quite significantly with the applied magnetic field inclining more towards the $y$-axis, as can be seen from Figures 14-16 (b). In addition, as we can see from Figures 12-16, there are some unexpected oscillations for both velocity and magnetic field in the small interval $[0.8, \sqrt{3} / 2]$. It is believed that the acute angle of the cross-section of the triangular channel is the cause of these variations.

\section{Conclusion}

In this paper, DRBEM in conjunction with linear radial basis functions being adopted as the interpolation functions is applied in the MHD steady channel flow to obtain the numerical solution of the axial velocity and the induced magnetic field. Comparisons between the DRBEM solutions and other solutions including the exact solution and FEM solution are made. Through three numerical test examples with different geometries of the cross-section of a channel, it is shown that a high numerical accuracy can be achieved with the DRBEM with simplest constant elements. In addition, the results of more general cases with an inclined magnetic field with arbitrary inclination angles being applied are presented too; the versatility of the current DRBEM model enables us to discuss the effect of inclined magnetic field on the flow. Our computational results show that the influence of the inclination angle of the applied magnetic field is far stronger on the induced magnetic field $-B$ than on the axial velocity.

\section{Acknowledgments}

The first author was supported in part by an Australian Government Overseas Postgraduate Research Scholarship, a University of Wollongong Postgraduate Award 


\section{and a Guangxi University for Nationalities Key Grant \#00SXX00001.}

The authors would also like to gratefully acknowledge some very constructive suggestions from two anonymous referees; based on these suggestions we have revised the paper to its current form.

\section{References}

[1] C. A. Brebbia, J. C. F. Tells and L. C. Wrobel (eds.), Boundary element techniques (Springer, Berlin, 1984).

[2] T. R. Bridges and L. C. Wrobel, "A dual reciprocity formulation for elasticity problems with body forces using augmented thin plate splines", Comm. Numer. Methods Eng. 12 (1996) 209-220.

[3] I. L. Brunton and A. J. Pullan, "A semi-analytic boundary element method for parabolic problem", Eng. Anal. Boundary Elements 18 (1996) 253-264.

[4] C. J. Coleman, D. L. Tullock and N. Phan-Thien, "An effective boundary element method for inhomogeneous partial differential equations", ZAMP 42 (1991) 730-745.

[5] J. Duchon, "Splines minimizing rotation-invariant semi-norms in Sobolev spaces", in Constructive theory of functions of several variables (Proc. Conf., Math. Res. Inst., Oberwolfach, 1976), Lecture Notes in Math. 571, (Springer, Berlin, 1977) 85-100.

[6] L. R. T. Gardner and G. A. Gardner, "A two dimensional bi-cubic B-spline finite element: used in a study of MHD-duct flow", Comput. Methods Appl. Mech. Eng. 124 (1995) 365-375.

[7] M. A. Golberg, "The method of fundamental solutions for Poisson's equation", Eng. Anal. Boundary Elements 16 (1995) 205-213.

[8] M. A. Golberg, C. S. Chen and S. Karur, "Improved multiquadric approximation for partial differential equations", Eng. Anal. Boundary Elements 18 (1996) 9-17.

[9] R. R. Gold, "Magnetohydrodynamic pipe flow, part. I", J. Fluid Mech. 13 (1962) 505-512.

[10] M. Itagaki and C. A. Brebbia, "Generation of higher order fundamental solutions to the twodimensional modified Helmholtz equation”, Eng. Anal. Boundary Elements 11 (1993) 87-90.

[11] A. D. Kerr, "An extension of the Kantorovich method", Quart. Appl. Math. 26 (1968) 219.

[12] H.-W. Liu, "Numerical modeling of the propagation of ocean waves", Ph. D. Thesis, University of Wollongong, Australia, 2001.

[13] H.-W. Liu, S.-P. Zhu and T. R. Marchant, "A perturbation DRBEM model for weakly nonlinear wave runups around islands", accepted in principle.

[14] W. R. Madych and S. A. Nelson, "Multivariate interpolation and conditionally positive definite functions II", Math. Comp. 54 (1990) 211-230.

[15] D. Nardini and C. A. Brebbia, A new approach to free vibration analysis using boundary elements (Comput. Mech., Southampton, and Springer, Berlin, 1982).

[16] A. C. Neves and C. A. Brebbia, "The multiple reciprocity boundary element method in elasticity: A new approach for transforming domain integrals to the boundary", Int. J. Numer. Methods Eng. 31 (1991) 709-727.

[17] A. J. Nowak and C. A. Brebbia, "The multiple-reciprocity method. A new approach for transforming BEM domain integrals to the boundary", Eng. Anal. Boundary Elements 6 (1989) 164-168.

[18] A. J. Nowak and C. A. Brebbia, "Solving Helmholtz equation by multiple reciprocity method", in Computer and experiments in fluid flow (eds. G. M. Carlomagno and C. A. Brebbia), (Comput. Mech., Southampton, 1989) 265-270.

[19] P. W. Partridge and C. A. Brebbia, "Computer implementation of the BEM dual reciprocity method for the solution of Poisson-type equations", Software Engrg. Workstations 5 (1989) 199-206. 
[20] P. W. Partridge, C. A. Brebbia and L. C. Wrobel, The dual reciprocity boundary element method (Comput. Mech., Southampton, and Elsevier Appl. Sci., London, 1992).

[21] P. W. Partridge and L. C. Wrobel, "The dual reciprocity method for spontaneous ignition", Int. J. Numer. Methods Eng. 30 (1990) 953-963.

[22] M. J. D. Powell, "The uniform convergence of thin plate splines in two dimensions", in Univ. of Cambridge Numer. Anal. Report DAMTP 1993/NA 16.

[23] M. J. D. Powell, "The theory of radial basis function approximation in 1990", in Advances in numerical analysis, Vol. II (Lancaster, 1990), (Oxford Univ. Press, New York, 1992) 105-210.

[24] J. A. Shercliff, "Steady motion of conducting fluid in pipes under transverse magnetic fields", Math. Proc. Cambridge Philos. Soc. 49 (1953) 136-144.

[25] B. Singh and J. Lal, "Magnetohydrodynamic axial flow in a triangular pipe under transverse magnetic field", Indian J. Pure Appl. Math. 9 (1978) 101-115.

[26] B. Singh and J. Lal, "MHD axial flow in a triangular pipe under transverse magnetic field parallel to a side of the triangle", Indian J. Tech. 17 (1979) 184-189.

[27] B. Singh and J. Lal, "Finite element method in magnetohydrodynamic channel flow problems", Int. J. Numer. Methods Eng. 18 (1982) 1104-1111.

[28] B. Singh and J. Lal, "Finite element method for MHD channel flow with arbitrary wall conductivity”, J. Math. Phys. Sci. 18 (1984) 501-516.

[29] B. Singh and J. Lal, "Finite element method for unsteady MHD flow through pipes with arbitrary wall conductivity", Int. J. Numer. Methods Fluids 4 (1984) 291-302.

[30] M. Tezer-Sezgin, "BEM solution of MHD flow in a rectangular duct", Int. J. Numer. Methods Fluids 18 (1994) 937-952.

[31] M. Tezer-Sezgin and S. Dost, "Boundary element method for MHD channel flow with arbitrary wall conductivity", Appl. Math. Modeling 18 (1994) 429-436.

[32] M. Tezer-Sezgin and S. Dost, "Boundary element solution of inhomogeneous modified Helmholtz equation", Scientia Iranica 1 (1994) 157-166.

[33] M. Tezer-Sezgin and S. Koksal, "Finite element method for solving MHD flow in a rectangular duct", Int. J. Numer. Methods Eng. 28 (1989) 445-459.

[34] Z. Wu and R. Shaback, "Local error estimates for radial basis function interpolation of scattered data", IMA J. Num. Anal. 13 (1993) 13-27.

[35] Y. L. Zhang and S.-P. Zhu, "On the choice of interpolation functions used in the dual-reciprocity boundary-element method", Eng. Anal. Boundary Elements 13 (1994) 387-396.

[36] R. Zheng, C. J. Coleman and N. Phan-Thien, "A boundary element approach for non-homogeneous potential problems", Comp. Mech. 7 (1991) 279-288.

[37] S.-P. Zhu and H.-W. Liu, "On the application of the multiquadric bases in conjunction with the LTDRM method to solve nonlinear diffusion equations", Appl. Math. Comput. 96 (1998) 161-175.

[38] S.-P. Zhu, H.-W. Liu and X.-P. Lu, "A combination of the LTDRM and the ATPS in solving linear diffusion problems", Eng. Anal. Boundary Elements 21 (1998) 285-289.

[39] S.-P. Zhu, P. Satravaha and X.-P. Lu, "Solving linear diffusion equations with the dual reciprocity method in Laplace space”, Eng. Anal. Boundary Elements 13 (1994) 1-10.

[40] S.-P. Zhu and Y. L. Zhang, "Improvement on dual reciprocity boundary element method for equations with convective terms", Comm. Numer. Methods Eng. 10 (1994) 361-371. 